\title{
Avaliação de Ambiente Virtual de Aprendizagem com o uso do SUS - System Usability Scale
}

\author{
Rafael Marimon Boucinha, PGIE/ UFRGS, rafael@ieduca.com.br \\ Liane Margarida Rockenbach Tarouco, PGIE/ UFRGS, liane@ penta.ufrgs.br
}

\begin{abstract}
Resumo: A presente pesquisa teve como objetivo avaliar a usabilidade do Sistema Interativo Ouro Moderno com a aplicação do questionário SUS - System Usability Scale. Através dos 155 questionários respondidos foi possível verificar o atendimento dos critérios: facilidade de aprendizagem do sistema $(76,05)$, eficiência do sistema $(76,45)$, baixo nível de inconsistências $(74,68)$ e satisfação dos usuários $(77,85)$. O Score SUS $(77,52)$ possibilitou avaliar, através de uma métrica, que o Sistema Interativo Ouro Moderno atende exigências de usabilidade dos empregados do Grupo CEEE. permitindo identificar oportunidades de melhoria do sistema, as quais, contribuirão para melhor usabilidade.
\end{abstract}

Palavras-chave: Usabilidade. Avaliação de Sistemas. Qualidade.

Abstract: The current survey has the objective of measuring the "Sistema Interativo Ouro Moderno" usability using the SUS Survey (System Usability Scale). Through 155 answered questionaires it was possible to verify the achievement of several criteria, as: ease of learning system $(76,05)$, system efficiency $(76,45)$, low level of inconsistencies $(74,68)$, and user satisfaction $(77,85)$. The SUS Score $(77,52)$ turns possible to evaluate, considering an especific metrical, that the "Sistema Interativo Ouro Moderno" achieves the usability requirements of Grupo CEEE employees, allowing to idtentify oportunities to improve the system.

Keywords: Usability. Measure Systems, Quality.

\section{Introdução}

À medida que as empresas estão identificando o papel da Tecnologia da Informação como diferencial competitivo, estão aprimorando o processo de aquisição de softwares e sistemas correlatos.

Para ser aceito pelo mercado, não basta um sistema atender requisitos de ordem técnica e as funcionalidades para as quais foi projetado. Os usuários exigem que seja fácil de utilizar e rapidamente aprendizado. Desta forma, a análise da IHC - Interface Homem Computador e a usabilidade de um sistema tem se destacado como fatores decisivos para definir a aquisição de sistemas.

A presente pesquisa refere-se a um Ambiente Virtual de Aprendizagem, o qual foi contratado pelo Grupo CEEE, com intuito de atender um maior número de colaboradores e reduzir os custos relacionados aos treinamentos corporativos, tais como transporte e diárias.

Considerando a necessidades de contratação de 4.000 vagas para os cursos básicos de informática, optou-se pela contratação inicial de 250 vagas, por meio de um Projeto Piloto com o propósito de avaliar a qualidade do sistema fornecido pela empresa Ouro Moderno.

Diante do exposto, tornou-se necessária uma avaliação objetiva referente à usabilidade do sistema, a qual poderá definir a possibilidade de um futuro contrato. Desta forma, 
apresenta-se o seguinte problema de pesquisa: Qual a avaliação dos usuários referente à usabilidade do Sistema Interativo Ouro Moderno?

O objetivo geral deste estudo foi: Avaliar a usabilidade do Sistema Interativo Ouro

Moderno. Para atender este objetivo, foi necessário estabelecer os seguintes objetivos específicos:

1. Avaliar a facilidade de aprendizagem do sistema;

2. Verificar a eficiência do sistema;

3. Identificar inconsistências do sistema;

4. Avaliar a facilidade de memorização;

5. Verificar a satisfação dos usuários;

6. Identificar oportunidades de melhoria do sistema.

\section{Usabilidade}

De acordo com Preece et al. (2003), usabilidade é um conceito chave na interação homem computador e enfatiza a preocupação em fazer sistemas que apresentem facilidade de aprendizagem e na utilização.

Para Nielsen (2003), usabilidade é um atributo de qualidade que avalia a facilidade de uso de uma interface, sendo definida por cinco componentes:

1. Capacidade de aprendizagem: a facilidade de utilizar o sistema pela primeira vez;

2. Eficiência: rapidez para executar as tarefas;

3. Memorização: o processo de lembrar como utilizar o sistema, após um tempo sem utilizar;

4. Erros: ausência de erros apresentados pelo sistema;

5. Satisfação: design agradável.

A usabilidade é um elemento-chave do design centrado no ser humano. "Humancentered design é uma filosofia de designer, um processo que leva em conta as capacidades humanas e limitações em cada estágio do processo de design" (SANDOR e HOLDEN, 2011, p.12).

Para Sordi e Meireles (2010), o nível de usabilidade de um sistema pode ser obtido avaliando-se características como a facilidade de aprendizagem da operação e a facilidade e eficiência de uso da interface.

Para Shackel (1986), ao utilizar o produto é que o usuário constrói sua percepção em relação ao mesmo. Para avaliação das tarefas sob o ponto de vista da usabilidade, o autor sugere quatro critérios:

1. Eficácia: avalia o desempenho de uma tarefa tendo como foco a análise da velocidade de execução e quantidade de erros;

2. Aprendizagem: avalia o desempenho desde a instalação do produto até o início do uso. Inclui a avaliação do tempo de treinamento necessário, do uso de suporte e da necessidade de novos treinamentos para reciclagem;

3. Flexibilidade: Avalia a capacidade de adaptação a novas tarefas além das já especificadas no sistema;

4. Atitude: Avalia o desempenho com relação ao conforto ou satisfação do usuário ao utilizar o sistema.

Bastien e Scapin (1993) desenvolveram um conjunto de critérios ergonômicos para construção de interfaces de sistemas. Estes autores propuseram oito critérios principais, dividido em subcritérios que complementam o conceito. Os oito critérios principais são:

1. Condução: refere-se aos meios disponíveis para aconselhar, orientar, informar, e conduzir o usuário na interação com o computador (mensagens, alarmes, rótulos, etc.). 
2. Carga de Trabalho: está relacionada aos elementos da interface, devido ao importante papel na redução da carga cognitiva e perceptiva do usuário, possibilitando aumento da eficiência do diálogo.

3. Controle Explícito: refere-se ao controle que os usuários tem sobre o processamento de suas ações pelo sistema.

4. Adaptabilidade: capacidade do software em se comportar conforme as necessidades e preferências do usuário.

5. Gestão de erros: compreende os mecanismos capazes de prevenir e reduzir erros causados pelo sistema, além de rotinas para facilitar sua correção quando os mesmos ocorrerem.

6. Consistência: o modo que os aspectos de interface são mantidos em contextos semelhantes, e se diferenciam em contextos distintos.

7. Significância do código: relação semântica entre os códigos e os itens ou ações às que eles se referem, demonstra a clareza com que os códigos e denominações são apresentados para o usuário.

8. Compatibilidade: é a relação entre as características dos usuários e as tarefas, saídas e entradas do sistema.

Considerando que a usabilidade é uma qualidade de uso, definida ou medida para um contexto em que um sistema é operado, Cybis (2003, p.4) afirma que "um sistema pode proporcionar boa usabilidade para um usuário experiente, mas péssima para novatos, ou vice e versa".

Sendo assim, o projetista de interfaces deve conhecer informações sobre quem será o usuário do sistema que está sendo projetado e o contexto que será utilizado.

Devido à importância do tema usabilidade, existem normas como a ISO que trata sobre este tema: "NBR ISO 9241: Requisitos Ergonômicos para Trabalho de Escritórios com Computadores Parte 11 - Orientações sobre Usabilidade".

\section{Normas técnicas relacionadas a usabilidade}

A ISO 9126 foi a primeira norma internacional a abordar o conceito de usabilidade, fornece modelo de propósito geral que define seis características e respectivas subcaracterísticas de qualidade de software: Funcionalidade, Confiabilidade, Usabilidade, Eficiência, Manutenibilidade e Portabilidade.

Nesta norma, a usabilidade é composta por 5 subcaracterísticas:

1. Inteligibilidade: facilidade do usuário em reconhecer a lógica de funcionamento do produto e sua aplicação.

2. Apreensibilidade: medida da facilidade de utilização do software pelo usuário.

3. Operacionalidade: medida da facilidade de operação do sistema.

4. Atratividade: evidencia a satisfação subjetiva do usuário durante o uso

5. Conformidade: atributos do software que fazem com que o software esteja de acordo com as normas, convenções ou regulamentações previstas para o tipo de aplicação.

Para a norma NBR ISO 9241-11, a usabilidade é considerada como sendo a "Medida na qual um produto pode ser usado por usuários específicos para alcançar objetivos específicos com eficácia, eficiência e satisfação em um contexto específico de uso" (ABNT, 2011, p.3).

A compreensão deste conceito torna-se necessário o conhecimento de outras definições apresentadas na NBR ISO 9241-11:

- Eficácia: acurácia e completude com as quais usuários alcançam objetivos específicos. 
- Eficiência: recursos gastos em relação à acurácia e abrangência com as quais usuários atingem objetivos.

- Satisfação: ausência do desconforto e presença de atitudes positivas para com o uso de um produto.

- Contexto de uso: usuários, tarefas, equipamento (hardware, software e materiais), e o ambiente físico e social no qual um produto é usado.

- Sistema de trabalho: sistema, composto de usuários, equipamento, tarefas e o ambiente físico e social, com o propósito de alcançar objetivos específicos.

A NBR ISO 9241-11 enfatiza que a usabilidade dependente sempre do contexto de uso e que o nível de usabilidade alcançado dependerá das circunstâncias específicas nas quais o produto é utilizado. A Figura 3, apresentada a seguir, demonstra a estrutura de usabilidade.

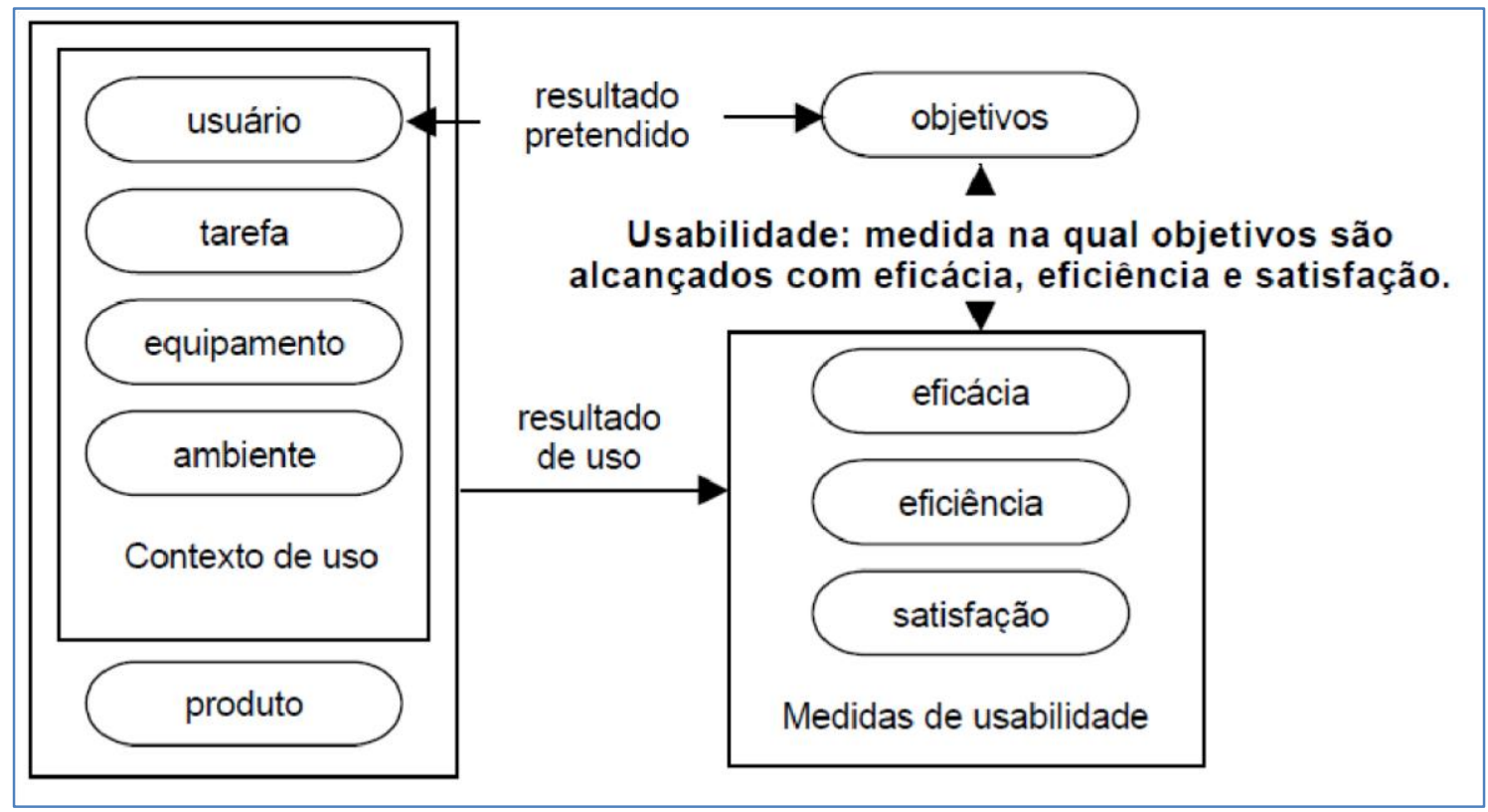

Figura 1: Estrutura de usabilidade.

Fonte: ABNT, 2011.

\section{Métodos para avaliação da usabilidade}

De acordo com Cybis (2003), é possível classificar os diferentes métodos utilizados para avaliar a usabilidade de um sistema de acordo com a técnica utilizada: (1) Técnicas Prospectivas, que buscam a opinião do usuário; (2) Técnicas Preditivas ou diagnósticas, que buscam prever os erros de projeto de interfaces sem a participação direta de usuários; e (3) Técnicas Objetivas ou empíricas, que utilizam a observação do usuário interagindo com o sistema. 

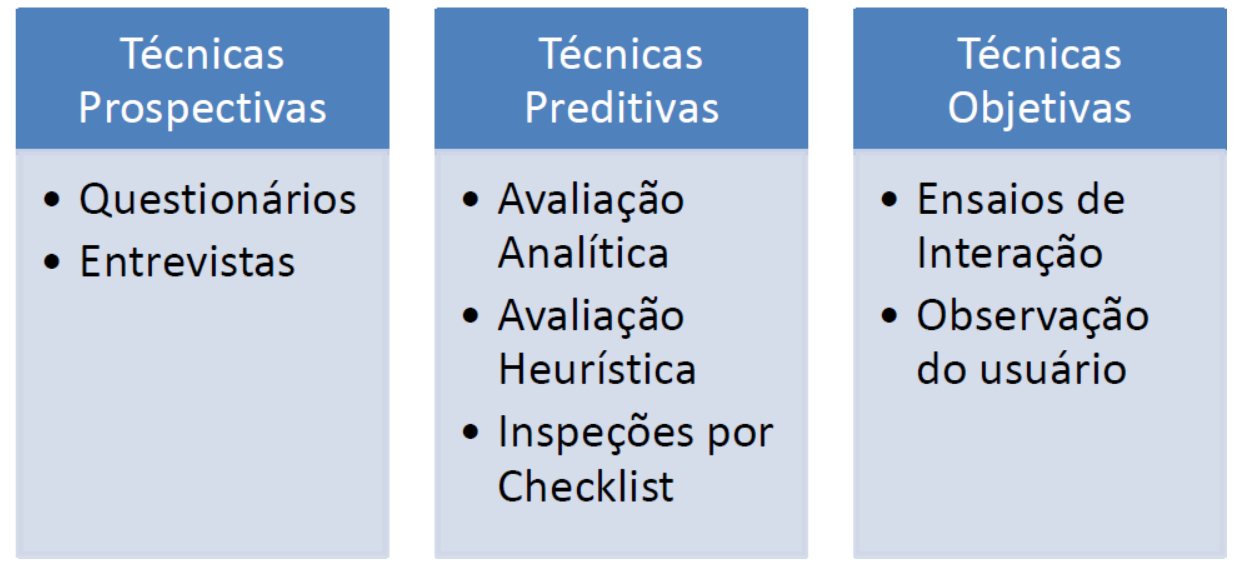

Figura 4: Técnicas para avaliação de usabilidade. Fonte:

Adaptado de Cybis, 2003.

\section{System Usability Scale (SUS)}

A escala SUS foi desenvolvida em 1986, por John Brooke, no laboratório da Digital Equipment Corporation, no Reino Unido. É um questionário composto por 10 itens, com 5 opções de respostas (SAURO, 2009).

SUS é uma tecnologia independente e já foi testado em hardware, software de consumo, sites, telemóveis, URAs e até o páginasamarela. Tornou-se um padrão da indústria com referências em mais de 600 publicações (SAURO, 2009, s/p).

O respondente do questionário SUS assinala sua resposta numa escala Likert que varia de Discordo totalmente a Concordo totalmente, observado na Figura 2.

\begin{tabular}{|c|c|c|c|c|c|}
\hline \multirow{2}{*}{$\begin{array}{l}\text { 1. I think that I would like to } \\
\text { use this system frequently }\end{array}$} & \multicolumn{4}{|l|}{$\begin{array}{l}\text { Strongly } \\
\text { disagree }\end{array}$} & \multirow[t]{2}{*}{$\begin{array}{l}\text { Strongly } \\
\text { agree }\end{array}$} \\
\hline & & & & & \\
\hline & 1 & 2 & 3 & 4 & 5 \\
\hline
\end{tabular}

Figura 2: Exemplo de questão do SUS.

Fonte: Brooke, 1986.

Considerando que este instrumento foi construído em língua inglesa, para preservar a validação do instrumento para língua portuguesa, na pesquisa de Tenório et al. (2011, p. 3) foi realizado um elaborado trabalho de tradução, onde todas as questões foram traduzidas para o português e, depois, um tradutor profissional realizou a tradução para língua inglesa. A tradução reversa teve como resultado um texto muito próximo do original demonstrando que o significado e estrutura do texto foram preservados na versão em português, por este motivo foi utilizada esta versão na pesquisa.

O resultado da SUS é a soma da contribuição individual de cada item. Para os itens ímpares deve-se subtrair 1 à resposta do usuário, ao passo que para os itens pares o score é 5 menos a resposta do usuário. Depois de obter o score de cada item, somam-se os scores e multiplica-se o resultado por 2,5 (BROOKE, 1986). Desta forma, o resultado obtido será um índice de satisfação do utilizador (que varia de 0 a 100).

De acordo com Sauro (2009), a pontuação SUS média dos 500 estudos que realizou foi de 68 pontos e Bangor et al. (2009) relatam que a média de 70 pontos tem se mantido em diferentes aplicações da SUS. O Quadro 5 apresenta uma síntese dos estudos de Bangor et al. (2009). 


\begin{tabular}{|l|l|l|l|}
\hline \hline Interface Type & Total Count & Count for this study & Total Mean Score \\
\hline Web & $1433(41 \%)$ & $317(33 \%)$ & 68.2 \\
\hline Cell phones & $593(17 \%)$ & $372(39 \%)$ & 65.9 \\
\hline IVR & $573(17 \%)$ & $228(23 \%)$ & 72.4 \\
\hline GUI & $250(7 \%)$ & $12(1 \%)$ & 76.2 \\
\hline Hardware & $237(7 \%)$ & $0(0 \%)$ & 71.8 \\
\hline TV & $185(5 \%)$ & $35(4 \%)$ & 67.8 \\
\hline Total & 3463 & 964 & 69.5 \\
\hline
\end{tabular}

Quadro 1: Score médio obtido com o SUS em diferentes interfaces.

Fonte: Bangor et al. (2009)

De acordo com Tenório et al. (2011) é possível reconhecer os componentes de qualidade indicados por Nielsen nas questões do SUS:

- Facilidade de aprendizagem: 3, 4, 7 e 10;

- Eficiência: 5, 6 e 8;

- Facilidade de memorização: 2;

- Minimização dos erros: 6;

- Satisfação: $1,4,9$.

\section{Material e Métodos}

Gil (2010) propõe que as pesquisas podem ser classificadas considerando seus objetivos e os procedimentos e técnicas utilizadas, enquanto Vergara (2010) afirma que a classificação da pesquisa é realizada em relação aos fins e aos meios utilizados.

Considerando o objetivo geral da presente pesquisa, é possível classificá-la como uma pesquisa descritiva, pois sua meta é descrever a avaliação de usabilidade do Sistema Interativo Ouro Moderno. De acordo com Gil (2010, p.28), "São incluídas neste grupo as pesquisas que têm por objetivo levantar as opiniões, atitudes e crenças de uma população".

No que se refere aos procedimentos e técnicas utilizados para a pesquisa, foi utilizado um questionário para obter a avaliação dos alunos em relação ao sistema utilizado, enquadrando a presente pesquisa como um levantamento.

As pesquisas deste tipo caracterizam-se pela interrogação direta das pessoas cujo comportamento se deseja conhecer. Basicamente, procede- se à solicitação de informações a um grupo significativo de pessoas acerca do problema estudado para, em seguida, mediante análise quantitativa, obteremse as conclusões correspondentes aos dados coletados (GIL, 2010, p.35).

Como o estudo foi realizado em apenas uma empresa e com um determinado Ambiente Virtual de Aprendizagem, a pesquisa enquadra-se como um estudo de caso.

De acordo com estudos de Tullis e Stetson (2004), para obter um resultado válido em teste de usabilidade, é necessário uma amostra entre 12 a 14 usuários.

Neste estudo, o questionário de pesquisa foi disponibilizado para todos participantes do curso. De acordo com Gil (2010, p.35), "Quando o levantamento recolhe informações de todos os integrantes do universo pesquisado, tem-se um censo", de maneira que esta pesquisa foi censitária.

Como limitação da presente pesquisa, sabe-se que os resultados não podem ser generalizados por se tratar de um estudo de caso, ou seja, os resultados e conclusões aplicam-se somente à empresa e ao sistema estudados.

Como o Sistema Interativo Ouro Moderno é um produto pronto, entende-se que avaliações de usabilidade preditivas não seriam adequadas para este caso. A ausência de especialistas na área de usabilidade no Grupo CEEE e a falta de recursos financeiros fez com que fosse descartado o uso de Técnicas Objetivas. 
Para a escolha da Técnica Preditiva a ser utilizada, foi considerado o fato dos alunos que utilizariam o Sistema Interativo Ouro Moderno estarem dispersos em todo Estado do Rio Grande do Sul. Desta forma, a utilização de questionário para esta pesquisa evitaria custos em deslocamentos para entrevistas e, ainda, possibilitaria atingir um grande número de usuários.

O Questionário SUS foi escolhido como instrumento para Avaliação de Usabilidade deste estudo por ser um instrumento gratuito e, devido ao pequeno número de questões, fato que poderia facilitar a aderência à pesquisa.

Por fim, outro fator relevante para a escolha do SUS foi a necessidade de um instrumento objetivo para decidir uma futura contratação do Sistema Interativo Ouro Moderno, e a escala escolhida apresenta uma métrica para avaliação da Usabilidade.

Para facilitar a coleta de dados, foi disponibilizado através do Moodle o questionário SUS (System Usability Scale) para os 253 alunos que realizaram um dos cursos da empresa Ouro Moderno.

\section{Resultados e discussão}

Embora todos os alunos tenham sido convidados a avaliar o Sistema, a participação na presente pesquisa era voluntária. Foram efetivadas 253 matrículas no Sistema. Contudo, observou-se que apenas $76,68 \%$ dos matriculados concluíram seu curso.

Cabe salientar que os dados coletados não permitem inferir acerca dos motivos relacionados à desistência de 23,32\% dos matriculados.

Tabela 1: Número de alunos por etapa do curso.

\begin{tabular}{lc}
\hline Etapa & $\mathbf{N}^{\mathbf{0}}$ \\
\hline Matriculados & 253 \\
Concluintes & 194 \\
Respondentes (SUS) & 155 \\
\hline
\end{tabular}

Fonte: Elaborado a partir dos dados da pesquisa.

Considerando a média de 70 pontos obtida nos estudos de Bango (2009), podemos observar no Gráfico 1, que apenas o item 4 não atingiu esta média: Eu acho que precisaria do apoio de um suporte técnico para ser possível usar este sistema. No entanto, se for utilizada a média relatada por Sauro (2012) de 68, nem mesmo este item ficaria abaixo da média.

Conforme relato dos profissionais que acompanharam o curso, foi possível observar que existiam algumas dificuldades em relação ao Sistema, bem como falhas técnicas relacionadas à configuração de hardware e de rede, que prejudicaram a utilização do sistema.

Nielsen (1995), ao abordar o tema Avaliação Heurística, afirma que todo sistema deve fornecer um manual de ajuda. Observou-se que o Sistema Interativo Ouro Moderno não possui documentação de ajuda. Quando o usuário tem dúvidas, o mesmo deve acessar um ícone e enviar uma mensagem para o tutor.

Cabe destacar que em nenhum momento o sistema apresenta um manual sobre utilização da plataforma. É possível que a disponibilidade de manuais de ajuda auxilie na redução da necessidade de suporte técnico on-line. 


\section{Resultados}

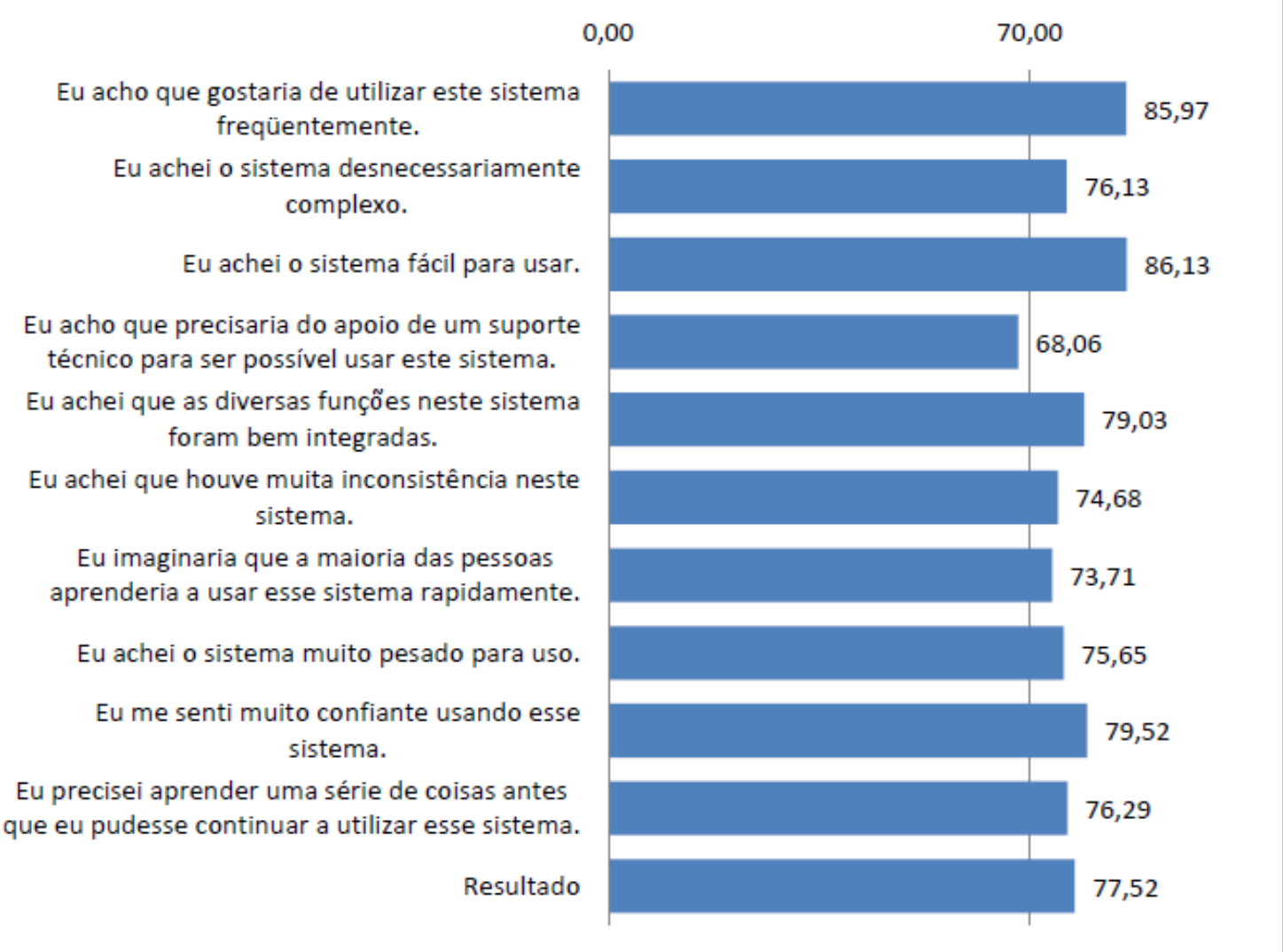

Gráfico 1: Resultados do SUS.

Fonte: Construído a partir dos dados da pesquisa.

Para verificar os objetivos específicos propostos para a presente pesquisa, foi utilizada a relação proposta por Tenório et al. (2011) entre os componentes de qualidade indicado por Nielsen e as questões do SUS:

- Avaliar a facilidade de aprendizagem do sistema: a facilidade de aprendizagem está representada nas 3, 4, 7 e 10 do SUS. A média do resultado destas questões é 76,05, portanto podemos concluir que os usuários apresentaram facilidade de aprender a utilizar o sistema.

- Verificar a eficiência do sistema: os itens 5, 6 e 8 estão relacionados a eficiência do Sistema. Analisando a média destas questões obtivemos 76,45, desta forma os usuários consideram o sistema eficiente.

- Identificar inconsistências do sistema: as inconsistências ou minimização dos erros são medidas através da questão 6. Neste item, o score SUS foi 74,68.

- Avaliar a facilidade de memorização: a facilidade de memorização é avaliada pela questão 2, o score de 76,13 demonstra satisfação em relação a este item.

- Verificar a satisfação dos usuários: a satisfação dos usuários está representada pelos itens: 1, 4, 9. A média destas questões foi 77,85.

- Identificar oportunidades de melhoria do sistema: Embora a usabilidade do Sistema Interativo Ouro Moderno tenha recebido uma boa avaliação por parte dos usuários, foi possível identificar alguns pontos que precisam ser aprimorados: O sistema deveria fornecer um manual de uso on-line, conforme propõe Nilsen; As avaliações de conhecimento e feedbacks podem ser 
aperfeiçoadas; Aprimorar o sistema disponibilizando uma opção para hardwares mais antigos e conexões mais lentas.

Observou-se, ainda, a influência de fatores externos ao sistema, cuja presença prejudicou seu uso. Embora estes itens não estivessem previstos nos objetivos específicos nesta pesquisa, devem ser objeto de atenção dos profissionais do Grupo CEEE. Desta forma verificou-se a necessidade de

- Verificar requisitos de hardware e software antes do início do curso; e

- Verificar condições de rede necessárias para acesso ao curso.

\section{Considerações Finais}

O retorno dos 155 questionários do SUS - System Usability Scale permitiu, por meio de uma métrica, afirmar que o Sistema Interativo Ouro Moderno atende aos critérios: facilidade de aprendizagem do sistema (76,05), eficiência do sistema (76,45), facilidade de memorização $(76,13)$ baixo nível de inconsistências $(74,68)$ e satisfação dos usuários $(77,85)$. Com estes resultados, foram atendidos os objetivos específicos: "1. Avaliar a facilidade de aprendizagem do sistema"; "2. Verificar a eficiência do sistema"; "3. Identificar inconsistências do sistema"; "4 Avaliar a facilidade de memorização" e "5. Verificar a satisfação dos usuários".

O Score SUS do Sistema Interativo Ouro Moderno de 77,52 demonstra que o sistema atende exigências de usabilidade dos empregados do Grupo CEEE. Embora a usabilidade tenha sido avaliada de forma positiva, este estudo permitiu identificar oportunidades de melhoria do sistema, as quais contribuirão para uma maior usabilidade.

O objetivo "6. Identificar oportunidades de melhoria" foi atendido com a sugestão da criação de um manual de uso on-line, aperfeiçoamento do sistema de avaliações feedbacks, e a criação de uma versão para hardwares mais antigos.

Embora não tenha sido proposto como objetivo específico, o estudo de usabilidade do sistema permitiu a visualização de oportunidades de melhoria no Grupo CEEE, indicando que é necessário analisar os pré-requisitos de hardware, de software e de rede antes de disponibilizar outros cursos utilizando o Sistema Interativo Ouro Moderno.

Por fim, a partir da exposição anterior, demonstrou-se que esta pesquisa atingiu plenamente os objetivos propostos. Conforme acordado, os resultados da pesquisa de usabilidade foram entregues à empresa para aperfeiçoamento do Sistema Interativo Ouro Moderno. Desta forma, sugere-se a realização de um novo estudo após as alterações do Sistema, utilizando o questionário SUS para, com a mesma métrica, comparar os resultados com este estudo inicial.

\section{REFERÊNCIAS}

ABNT. Associação Brasileira de Normas Técnicas. NBR 9241: Requisitos Ergonômicos para Trabalho de Escritórios com Computadores - Parte 11 - Orientações sobre Usabilidade. 2011. Disponível em:

<http://www.inf.ufsc.br/ cybis/pg2003/iso9241-11F2.pdf>. Acesso em: 16 dez. 2012. BANGOR, Aaron, KORTUM, Philip, e MILLER, James. Determining What Individual SUS Scores Mean: Adding an Adjective Rating Scale. Journal of Usability Studies, v.4, n.3, p.114-123, maio 2009. Disponível em:

<http://www.upassoc.org/upa_publications/jus/2009may/JUS_Bangor_May20 09.pdf>. Acesso em: 05 nov. 2012.

BASTIEN, J.M.C., e SCAPIN, D.L. Human factors criteria, principles, and recommandations for HCI: methodological and standatdisation issues. França: INRIA, 1993. 
BROOKE, J. SUS - A quick and dirty usability scale. 1986. Disponível em $<$ http://www.usabilitynet.org/trump/documents/Suschapt.doc $>$. Acesso em: $12 \mathrm{dez}$. 2012.

CYBIS, Walter de Abreu. Engenharia de Usabilidade: Uma Abordagem Ergômica. 2003. Disponível em:

<http://www.unoescsmo.edu.br/poscomp/cybis/Apostila_v51.pdf>. Acesso em: 05 out. 2012.

GIL, Antonio Carlos. Como elaborar projetos de pesquisa. 5 ed. São Paulo: Atlas, 2010.

ISO. International Organization for Standardization. IEC 9126: Software Engineering - Product Quality - Part 1: Quality Model. 2011. Disponível em:

<http://www.sqa.net/iso9126.html>. Acesso em: 18 dez.2012.

NIELSEN, J. Ten Usability Heuristics. 1994. Disponível em

<http://www.nngroup.com/articles/ten-usability-heuristics/>. Acesso em: 01 out. 2012. NIELSEN, J. Usability 101: Introduction to usability 2003. Disponível em:

<http://www.useit.com/alertbox/20030825.html>. Acesso em: 05 nov. 2012.

NORMAN, Donald A. Design of Everyday Things. 1 ed. New York: Basic Books, 2002. 272p.

PREECE, J. et al. A Guide to Usability: human factors in computing. Reino Unido: Addison Wesley, 1993. 144p.

SAURO, Jeff. Measuring Usability With The System Usability Scale

(SUS). 2009. Disponível em: <http://www.measuringusability.com/sus.php>. Acesso em: 09 out. 2012.

SHACKEL, B. Ergonomics in design for usability. In: HCI 86 Conference on People and Computer. New York: Cambridge University Press, 1986.

SORDI, José Osvaldo, MEIRELES, Manuel. Administração de Sistemas de informação: uma abordagem interativa. São Paulo: Saraiva, 2010.

TENÓRIO, Josceli Maria et al. Desenvolvimento e Avaliação de um Protocolo

Eletrônico para Atendimento e Monitoramento do Paciente com Doença Celíaca.

2011. Disponível em:

<http://www.sbis.org.br/cbis11/arquivos/693.pdf>. Acesso em: 11 ago. 2012.

TULLIS, Thomas S., e STETSON, Jacqueline N. A Comparison of Questionnaires for Assessing Website Usability. 2004. Disponível em:

<http://home.comcast.net/ tomtullis/publications/UPA2004TullisStetson.pdf>. Acesso em: 18 nov. 2012.

VERGARA, Sílvia. Projetos e relatórios de pesquisa em administração. 12 ed. São Paulo: Atlas, 2010. 